\title{
CHAPTER
}

\section{Introduction to spinal cord injury as clinical pathology}

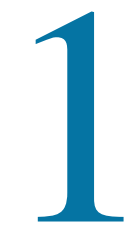

\author{
Simonetta Papa, PhD ${ }^{1}$, Emanuele Mauri, PhD ${ }^{2}$, Filippo Rossi, PhD ${ }^{1,3}$, \\ Giuseppe Perale, $\mathrm{PhD}^{1,4,5}$, Pietro Veglianese, $\mathrm{PhD}$ \\ ${ }^{1}$ Department of Neuroscience, IRCCS Istituto di Ricerche Farmacologiche "Mario Negri", Milan, \\ Italy; ${ }^{2}$ Department of Engineering, Tissue Engineering and Chemistry for Engineering Unit, \\ Università Campus Bio-Medico di Roma, Rome, Italy; ${ }^{3}$ Department of Chemistry, Materials and \\ Chemical Engineering "Giulio Natta”, Milan, Italy; ${ }^{4}$ Faculty of Biomedical Sciences of the \\ University of Southern Switzerland, Lugano, Switzerland; ${ }^{5}$ Ludwig Boltzmann Institute for \\ Experimental and Clinical Traumatology, Vienna, Austria
}

\section{Spinal cord anatomy}

The central nervous system, formed by brain and spinal cord, is the center in charge of reception, elaboration, and information transmission. The spinal cord comprises a bundle of neurons and has its place in the spinal canal that protects it. In humans, it has a cylindrical form, with $8-10 \mathrm{~mm}$ of medium diameter, starts at the base of the skull, but does not occupy the vertebral canal for his entire length, ending in the firsts lumbar vertebras. The spinal column and the spinal cord are divided into five regions: cervical, thoracic, lumbar, sacral, and coccyx (Fig. 1.1). From the spinal cord start the peripheral nerves, responsible for motor and sensory capabilities of the entire body. An external stimulus detected, for example, by the skin, is converted into chemical signals that are transported along the axons to the dorsal side of spinal cord and finally to the brain. In the brain, they are elaborated and the responsive motor signal goes back to the ventral side of the spinal cord and to the region that perceived the stimulus.

Three membranes, called meninges, of protective tissue cover the entire spinal cord. The external membrane has been named dura mater because of this strength, the intermediate one is the delicate arachnoid, which is thin and with web-like filaments, and the inner membrane is called pia mater. Between arachnoid and pia mater, there is a space filled with cerebrospinal liquid, and this gap contains arteries that supply blood to the spinal tissue: in case of trauma, there will be hemorrhaging.

\section{Internal organization}

The spinal cord is composed of two different types of tissue: the inner part appears grayish and is called gray matter; the outer instead is white and is called white matter (Fig. 1.2). White matter is subdivided into columns, each one containing neurons 


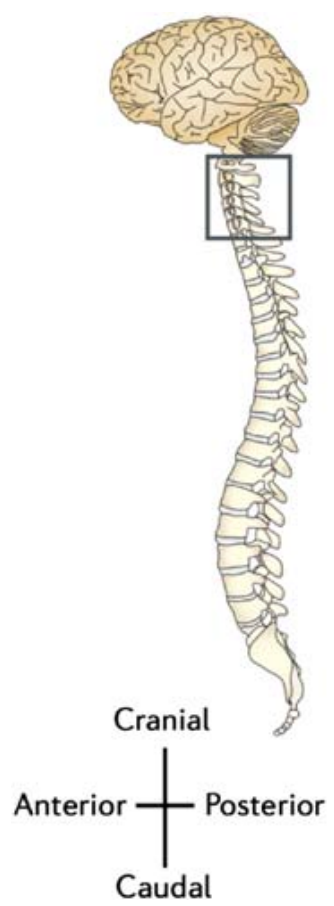

FIGURE 1.1

Spinal cord anatomy.

Reprinted with permission from Thuret S, Moon LDF, Gage FH. Therapeutic interventions after spinal cord injury.

Nat Rev Neurosci 2006; 7:628-43.

related to specific functions. The lateral columns include axons that travel from the cerebral cortex to contact spinal motor neurons. In the dorsal zone, sensibility information are carried up way to the brain. The ventral columns carry both ascending information about pain and temperature and descending motor information. Gray matter is divided into dorsal and ventral horns. The dorsal ones are for skin stimulus reception; the ventral ones receive proprioceptive fibers, and the middle part receives sensorial inputs from muscles and viscera. The muscle's motor activity is the result of the cooperation that takes place between different groups of neurons put in series: if one of them is damaged, the information circuit would be broken and the movement results impeded.

\section{Spinal cord injuries}

Spinal cord injury (SCI) is a condition that implies a loss of motor abilities and results to be one of the first causes of noncongenic disability in the world, with an annual incidence between 40 and 80 cases per million people. About two and a 


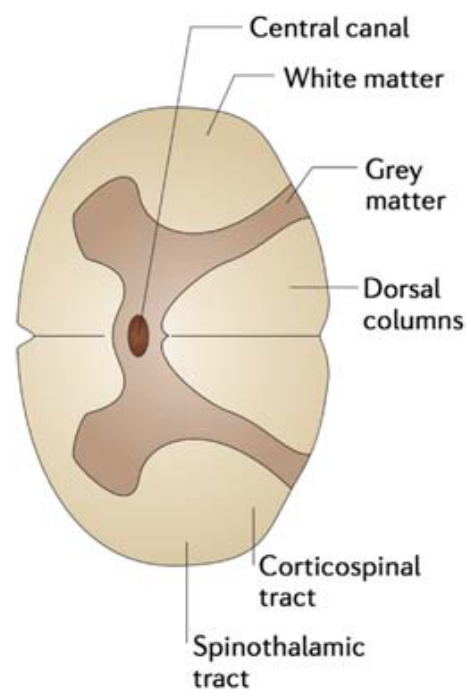

FIGURE 1.2

Spinal cord organization.

Reprinted with permission from Thuret S, Moon LDF, Gage FH. Therapeutic interventions after spinal cord injury.

Nat Rev Neurosci 2006;7:628-43.

half millions of the people worldwide are affected by SCI [2-4]. Most of them are male (82\% in the United States, $72.7 \%$ in India), and the mean age is 33 years, with a tendency to rise. The causes vary in the world differently from country to country and are mostly related to vehicular accidents, falls, violence, or sport activities. In addition, the incidence is region-dependent and visible in Fig. 1.3 [5]. The results of these traumatic events are immediate breakage, dislocation, or compression of one or more vertebras and afterward hemorrhage, inflammation, and infection contribute to worse the clinical situation. However, the latest statistics evidence on the increase of cases with nontraumatic origin (osteoporosis or a tumor) in the North America and European Union regions correlated to the progressively aging population. Due to the violence of the trauma, a high percentage $(15 \%-56 \%)$ of people victim of SCI dies before hospitalization. The ones who survived the trauma and the first $24 \mathrm{~h}$ had lower life expectancy compared to population without SCI, in dependence to the resulting condition (paraplegia, tetraplegia) and the capacity of the healthcare system.

\section{SCI consequences}

The most frequent SCI consequences are loss of sensibility and paralysis below the level of injury. Four cases are determined: paraplegia, tetraplegia, paraparesis, and tetraparesis (Fig. 1.4). Paraplegia is a syndrome that includes complete paralysis of lower limbs and sphincteric disorders; in tetraplegia are added also upper limbs 

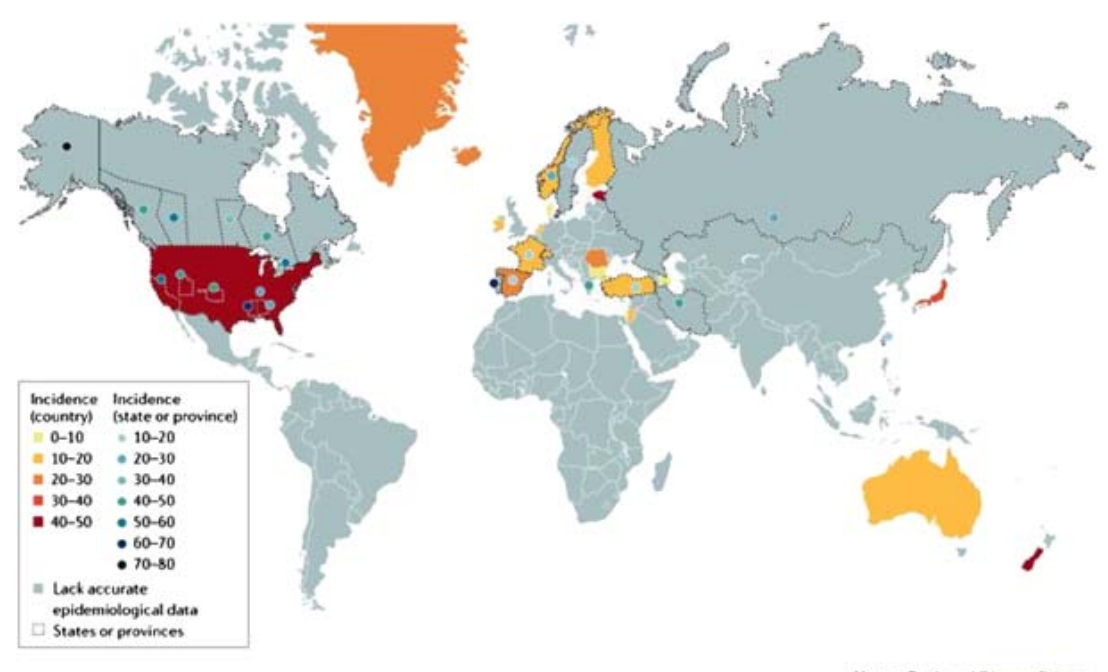

FIGURE 1.3

Relative annual incidences of countries, states/provinces, and regions. The red color scheme illustrates incidences of countries. The blue color scheme highlights incidences of states/provinces and regions (mil = million).

Reprinted with permission from Ahuja CS, Wilson JR, Nori S, Kotter MRN, Druschel C, Curt A, et al. Traumatic spinal cord injury. Nat Rev Dis Primers 2017;17018.

paralysis and, in the worst cases, autonomic nervous system's disorders. Paraparesis and tetraparesis are instead the incomplete paralysis of lower limbs or all of them.

The differences in outcomes are due to different injury levels along the vertebral column and by the lesion's completeness. Given the same neurological examination and findings, neurologists and physiatrists may not assign the same SCI level: neurologists define the level of injury as the first spinal segmental level that shows abnormal neurological loss.

In contrast, physiatrists or rehabilitation doctors tend to define the level of injury as the lowest spinal segmental level that is normal. For example, if a patient has normal C3 sensations and absent $\mathrm{C} 4$ sensations, a physiatrist would say the sensory level is $\mathrm{C} 3$ whereas a neurologist would call it a C4 injury level. A complete lesion implies the total and definitive inability of nervous information exchange between the two sides of the injury and therefore the loss of sensitive and motor abilities below the lesion level. In an incomplete lesion, some motor and sensitive capacities are maintained.

Consequences of SCI are as follows:

- Motion loss, with alteration of muscular tone and spastic contractions

- Skin sensibility loss

- Autonomic dysreflexia

- Urinary bladder loss of control 
Classification of spinal cord injury (SCl) severity using the American Spinal Injury Association (ASIA) Impairment Scale. The main categories of the Impairment Scale are as follows:

-A (complete): No motor or sensory function is preserved in the sacral segments S4-S5.

- B (incomplete): Sensory but not motor function is preserved below the neurological level and includes the sacral segments S4-S5.

- C (incomplete): Motor function is preserved below the neurological level, and more than a half of key muscles below the neurological level have a muscle grade of $<3$.

- D (incomplete): Motor function is preserved below the neurological level, and at least a half of key muscles below the neurological level have a muscle grade of $\geq 3$.

- E (normal): Motor and sensory functions are normal.

Extent of injury after damage to specific spinal segments is illustrated in the figure (see American Spinal Injury Association in Online links box for the complete standard neurological classification of $\mathrm{SCl})$.

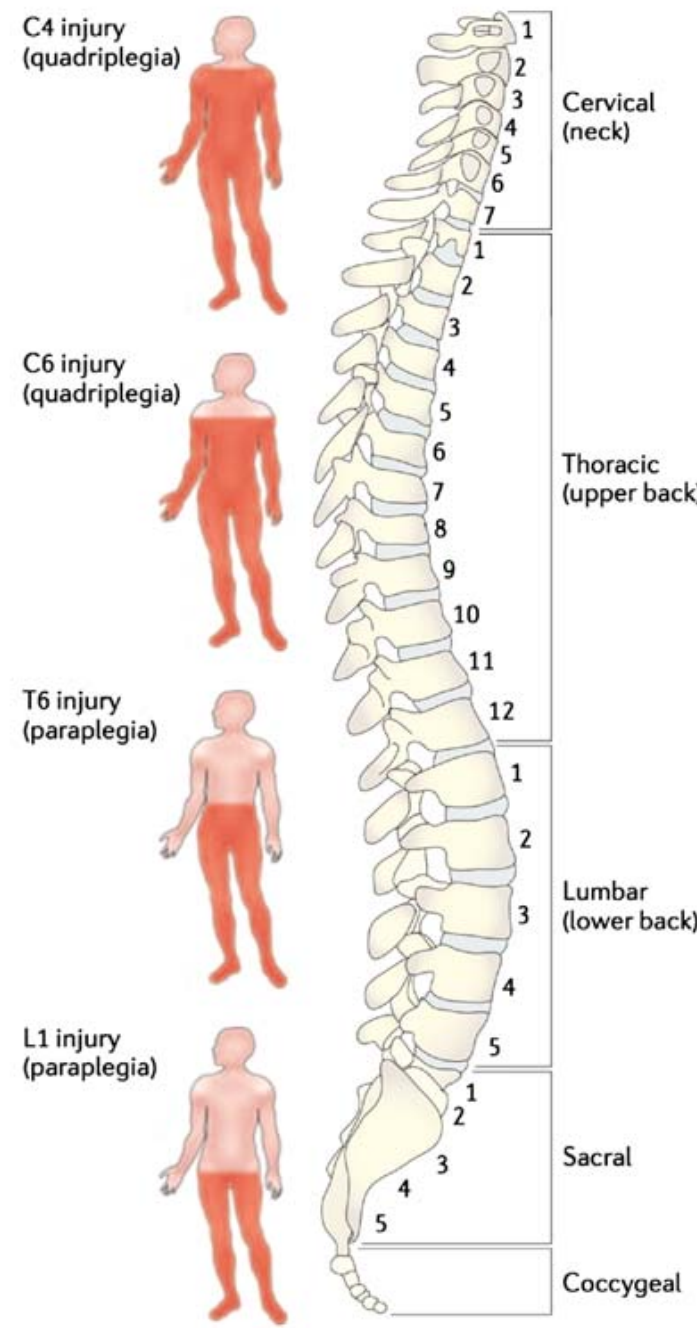

FIGURE 1.4

SCl levels.

Reprinted with permission from Thuret S, Moon LDF, Gage FH. Therapeutic interventions after spinal cord injury. Nat Rev Neurosci 2006; 7:628-43.

- Bowel loss of control

- Pain or burning sensation

- Breathing difficulties

- Circulatory problems 


\section{The ASIA impairment scale}

ASIA scale is a rating system used to define and describe the extent and severity of a patient's SCI and helps to determine future rehabilitation and recovery needs (Fig. 1.5). It is ideally completed within $72 \mathrm{~h}$ after the initial injury [7]. The patient's grade is based on how much sensation he can feel at multiple points on the body, as well as tests of motor function.

The scale has five degrees of seriousness:

$\mathbf{A}=$ Complete lack of motor and sensory function below the level of injury (including the anal area)

$\mathbf{B}=$ Some sensation below the level of the injury (including anal sensation)

$\mathbf{C}=$ Some muscle movement is spared below the level of injury, but $50 \%$ of the muscles below the level of injury cannot move against gravity

$\mathbf{D}=$ Most (more than 50\%) of the muscles that are spared below the level of injury are strong enough to move against gravity.

$\mathbf{E}=$ All neurologic function has returned.

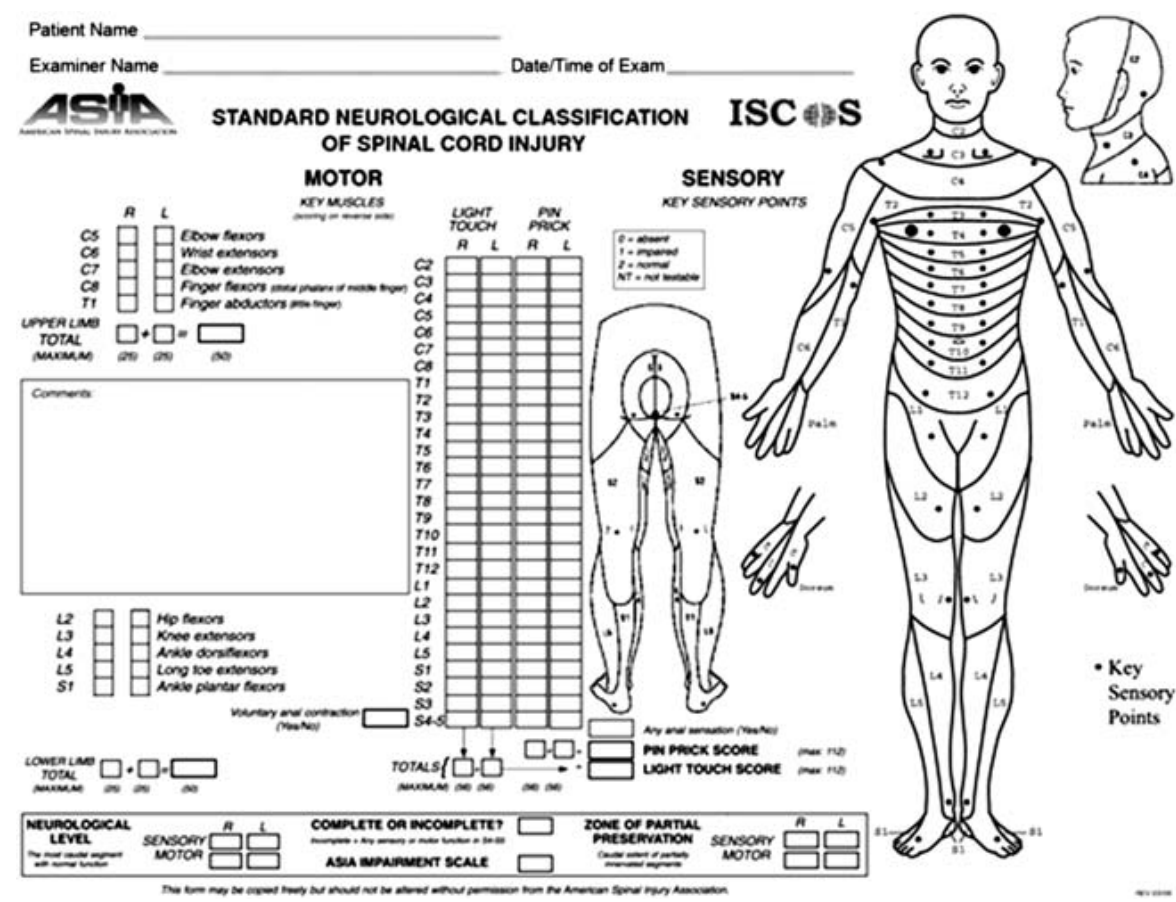

FIGURE 1.5

ASIA classification, used for the allocation of the neurological level and injury severity. 


\section{Prognosis}

When the SCI occurs, the spinal cord swells and some changes are seen in every system of the body. After days or weeks, the swelling begins to go down and some functioning may be regained. The improvement is not due to neuronal/axonal regeneration, but rather to hematoma reabsorption and reduction of the inflammation state. With many injuries, especially the incomplete ones, the patient may recover some functioning as late as 18 months after the injury, but usually the recovery ends in 6 months. Younger patients have major probabilities of motoric recovery, and two hypotheses attempt to explain this:

- Advanced age implies lower functional potential [8];

- Elder patients have more difficulty to translate neuronal recovery into physical activity [9].

\section{Physiopathology}

Three phases can be distinguished after SCI:

- Acute phase,

- Subacute phase,

- Chronic phase.

In the acute phase, starting immediately after the lesion, death of all types of cells (neurons, astrocytes, oligodendrocytes, and endothelia cells) occurs. Endothelial dying cells determine the breakage of arteries and hemorrhaging, with formation of edema near the injured region [10] (Fig. 1.6).

The subacute phase arises in few minutes from the injury and lasts for weeks. During this phase, usually patient conditions could be worsened by local inflammation state. Dead cells and the income and activation of microglia, macrophages, and lymphocytes modify the extracellular environment, intoxicating survived cells. When the inflammation diminishes, the chronic phase succeeds. A scar forms around the injury; it isolates and protects the damaged tissue but obstacles nerve regeneration. Near the scar states, a population of fibroblasts, activated macrophages and glial cells and often a cyst can be seen [12,13].

\section{Limited central nervous system regeneration}

Different from peripheral nervous system (PNS), the Central Nervous System (CNS) has no regeneration skills. Research studies $[13,14]$ demonstrate that the different behaviors of PNS and CNS are more associated to environmental condition than to cell's different physiology.

Inflammation and glial scar's proteins create an inhibitory environment and in the same time the regeneration associated genes deregulation extinguishes innate 


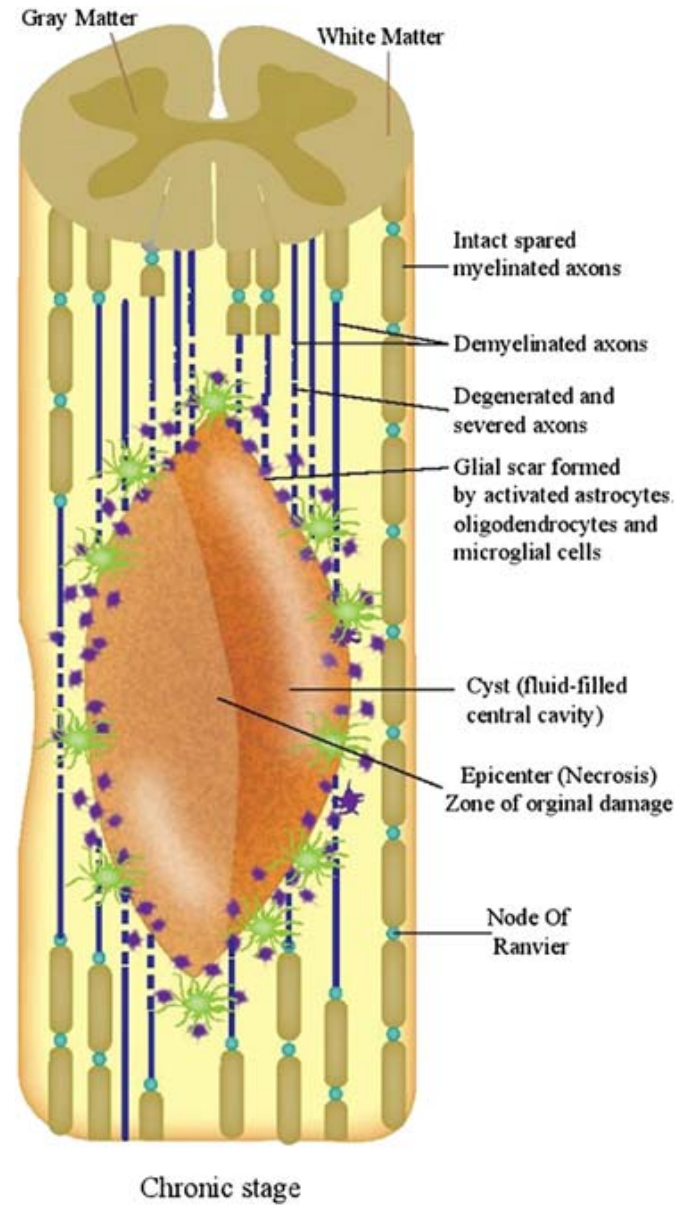

FIGURE 1.6

Spinal cord physiopathology after injury.

Reprinted with permission from Quadri SA, Farooqui M, Ikram A, Zafar A, Khan MA, Suriya SS, et al. Recent update on basic mechanisms of spinal cord injury. Neurosurg Rev 2018;7:1-17.

neuronal regeneration ability. Some days after the injury, astrocytes have morphological changes and become hypertrophic. They accumulate surrounding the lesion, incorporate microglia and oligodendrocytes, and link each other forming the scar. Microglia have a key role in recovery of CNS physical integrity and in closing up damaged brain blood barrier (BBB). Fixing BBB stops the income of unwanted peripheral cells and reduces cellular damage diffusion [15-17]. Nevertheless, glial scar produces axonal germination chemical inhibitors. 


\section{Role of inflammation in posttrauma recovery}

After primary lesion, the inflammatory state occurs: its activation is promoted by necrotic and apoptotic processes (Fig. 1.7). It comprises three steps:

1. In the initial phase, BBB breakage allows hemorrhaging and inflammatory cells arrival meanwhile nervous system's cells dye and lost their myelin cover. Microglia and astrocytes arrive from all CNS and try to restore tissue homeostasis $[18,19]$. Glial cells release proteins, called cytokines and chemokines, which can act both as proinflammatory (TNF- $\alpha$, tumor necrosis factor, interleukins IL-1 $\beta$ and IL-6) and as antiinflammatory factors [20,21]. With hemorrhage, macrophages and other immune system's cells easily enter into the system showing two different contributions to lesion's evolution.

On the one hand, they cause the secondary damage after SCI [10,22], and a treatment for limit or eliminate their presence seems to be useful in the recovery [23]; on the other hand, in the lesion site, they differentiate into M2

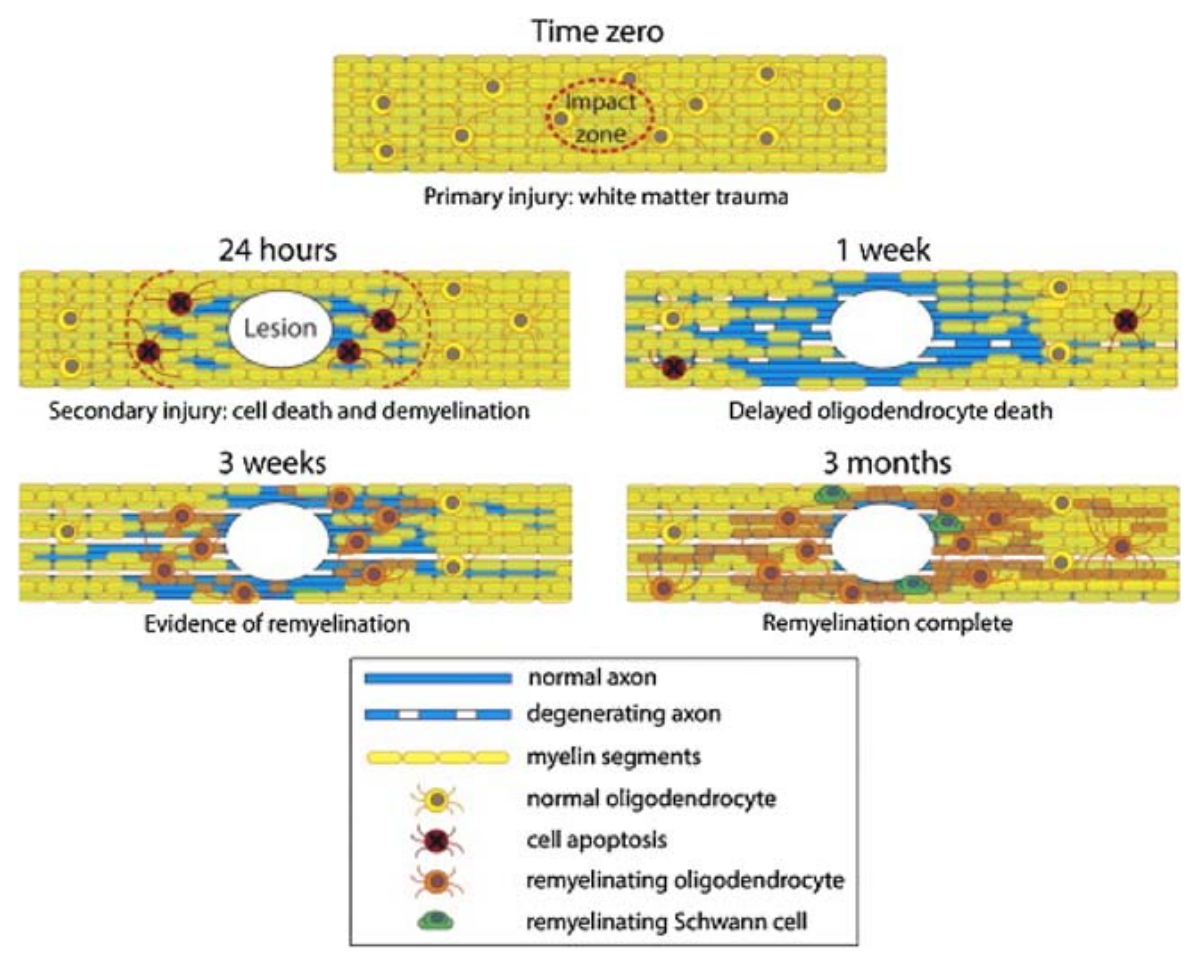

FIGURE 1.7

Primary and secondary injury.

Reprinted with permission from Plemel JR, Keough MB, Duncan GJ, Sparling JS, Yong VW, Stys PK, et al. Remyelination after spinal cord injury: is it a target for repair? Prog Neurobiol 2014;117:54-72. 
macrophages and emit antiinflammatory cytokines, arousing microglia deactivation $[24,25]$ and limiting damage diffusion.

2. Between 4 days and 2 weeks after injury, edema reduces and an increase of macrophages and microglia cells number can be observed [26,27]. Removing debris, macrophages create a cavity.

3. The third phase, in which there is a spinal cord volume contraction, is called reactive gliosis. Astrocytes become the first cell population [28] and form the glial scar [29].

\section{Role of microglia in SCI}

Microglial cells are a particular type of immune system's cells that arrive in CNS during fetal period and assume its defense from infections and diseases [31].

Discovered at the beginning of XX century by Rio-Hortega, they represent $10 \%-20 \%$ of adult CNS cells. Microglia have macrophage abilities, provide to CNS homeostasis by digesting apoptotic bodies and noxious substances, and react to endogenous (cytokines, altered levels of neurotransmitters, and proteins) and exogenous factors (viral membrane glycoproteins) [32].

In normal conditions, microglia are in deactivated form, with dendritic morphology for better environmental control. After activation stimulus, it rushes to the lesion site and assumes first amoeboid and later phagocytic form [33]. There are two possible phenotypes of activated microglia (Fig. 1.8):

- M1, proinflammatory, produces cytokines responsible of body defense reaction but even of new damage to healthy cells [34]. It has been demonstrated that it is possible to reproduce in vitro this phenotype treating microglia with different stimuli, such as LPS, a lipopolysaccharide forming bacterial protective membrane $[35,36]$.

- M2 is instead antiinflammatory and activated, for example, by IL-3 and IL-4. It helps injury recovery with enzymes that enhance phagocytosis and tissue's remodeling [34]. In vitro study confirmed that M2 microglia present no toxicity and ability to promote axonal growth [37].

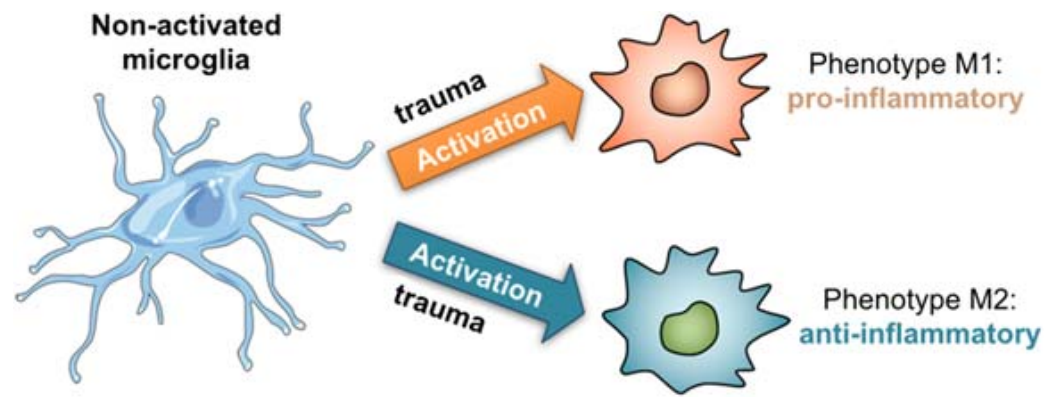

FIGURE 1.8

Microglia phenotypes. 


\section{References}

[1] Thuret S, Moon LDF, Gage FH. Therapeutic interventions after spinal cord injury. Nat Rev Neurosci 2006;7:628-43.

[2] Vismara I, Papa S, Rossi F, Forloni G, Veglianese P. Current options for cell therapy in spinal cord injury. Trends Mol Med 2017;23:831-49.

[3] Rossi F, Perale G, Papa S, Forloni G, Veglianese P. Current options for drug delivery to the spinal cord. Expert Opin Drug Deliv 2013;10:385-96.

[4] Veneruso V, Rossi F, Villella A, Bena A, Forloni G, Veglianese P. Stem cell paracrine effect and delivery strategies for spinal cord injury regeneration. J Control Release 2019;300:141-53.

[5] Singh A, Tetreault L, Kalsi-Ryan S, Nouri A, Fehlings MG. Global prevalence and incidence of traumatic spinal cord injury. Clin Epidemiol 2014;6:309-31.

[6] Ahuja CS, Wilson JR, Nori S, Kotter MRN, Druschel C, Curt A, et al. Traumatic spinal cord injury. Nat Rev Dis Primers 2017:17018.

[7] Herbison AE, Theodosis DT. Neurotensin-immunoreactive neurons in the rat medial preoptic area are oestrogen receptive. J Neuroendocrinol 1991;3:587-9.

[8] DiGiovanna AG. Human ageing: biological perspectives. New York, NY: McGraw Hill Companies; 2000.

[9] Jakob W, Wirz M, van Hedel HJ, Dietz V, Group E. Difficulty of elderly SCI subjects to translate motor recovery-“body function"-into daily living activities. J Neurotrauma 2009;26:2037-44.

[10] Donnelly DJ, Popovich PG. Inflammation and its role in neuroprotection, axonal regeneration and functional recovery after spinal cord injury. Exp Neurol 2008;209:378-88.

[11] Quadri SA, Farooqui M, Ikram A, Zafar A, Khan MA, Suriya SS, et al. Recent update on basic mechanisms of spinal cord injury. Neurosurg Rev 2018;7:1-17.

[12] B JH, N MD, K S, P W, M A, D D. Schwannosiss: role of gliosis and proteoglycan in human spinal cord injury. J Neurotrauma 2000;17:781-8.

[13] Chong MS, Woolf CJ, Turmaine M, Emson PC, Anderson PN. Intrinsic versus extrinsic factors in determining the regeneration of the central processes of rat dorsal root ganglion neurons: the influence of a peripheral nerve graft. J Comp Neurol 1996;370: 97-104.

[14] Yiu G, He Z. Glial inhibition of CNS axon regeneration. Nat Rev Neurosci 2006;7: 617-27.

[15] Reier PJ, Perlow MJ, Guth L. Development of embryonic spinal cord transplants in the rat. Brain Res 1983;312:201-19.

[16] Faulkner JR, Herrmann JE, Woo MJ, Tansey KE, Doan NB, Sofroniew MV. Reactive astrocytes protect tissue and preserve function after spinal cord injury. J Neurosci 2004;24:2143-55.

[17] Sofroniew MV. Molecular dissection of reactive astrogliosis and glial scar formation. Trends Neurosci 2009;32:638-47.

[18] Davalos A, Fisher M. Emerging therapies for cerebrovascular disorders. Stroke 2005; 36:208-10.

[19] Myer DJ, Gurkoff GG, Lee SM, Hovda DA, Sofroniew MV. Essential protective roles of reactive astrocytes in traumatic brain injury. Brain 2006;129:2761-72.

[20] Rice T, Larsen J, Rivest S, Yong VW. Characterization of the early neuroinflammation after spinal cord injury in mice. J Neuropathol Exp Neurol 2007;66:184-95. 